\title{
Challenges of Molecular Nanotechnology for Space Exploration
}

\author{
Thomas L. M'Kendree* \\ Raytheon, Fullerton, CA, 92646-3310 \\ Robert A. Freitas Jr. ${ }^{\dagger}$ \\ Institute of Molecular Manufacturing, Palo Alto, CA 94301 \\ Al Globus* \\ Computer Sciences Corporation, Moffett Field, CA, 94035 \\ M. Creon Levit ${ }^{\S}$ \\ NASA Ames Research Center, Moffett Field, CA 94035-1000 \\ and \\ C. David Sherrill** ${ }^{* *} \mathrm{Li}^{\dagger \dagger}$ and Ralph C. Merkle ${ }^{\dagger}$ \\ Georgia Institute of Technology, Atlanta, GA, 30332
}

\begin{abstract}
Molecular nanotechnology is the future capability to design and build systems to atomic precisions. This paper motivates the desire of molecular nanotechnology for space exploration with a review of potential benefits, and reviews relevant progress to date for molecular nanotechnology. It provides a framework of challenges that need to be resolved before molecular nanotechnology can be applied to space systems, with discussion on addressing those challenges. The primary focus is in the area of developing tools for automated design of molecular components, using molecular connector components as an example, and in developing tools for generating reaction pathways to fabricate molecular components that can integrate with tools for developing molecular components. The secondary focus is issues for the molecular fabrication system, including integration with the design of end product space systems.
\end{abstract}

\section{Nomenclature}

$\delta \quad=$ material density

$\sigma \quad=$ material strength

$\Delta \mathrm{V} \quad=$ change in velocity

\section{Introduction}

$\mathrm{M}$ olecular Nanotechnology, the emerging ability to design and build to atomic precision, offers significant potential to contribute to future space exploration efforts, including greater strength-to-weight materials,

\footnotetext{
* Principal Systems Engineer, Network Centric Systems, FU/676/X343, 92843-3310, Full Member.

${ }^{\dagger}$ Senior Research Fellow, 555 Bryant Street, Suite 354.

* Computer Scientist, Evolvable Systems Group, MS 269-3.

${ }^{\S}$ Research Scientist, Advanced Supercomputing Division, MS T27-A.

** Associate Professor, Chemistry \& Biochemistry, 770 State Street, 30332-0400.

${ }^{\dagger}$ Associate Professor, Materials Science and Engineering, 771 Ferst Drive, N.W, 30332-0245.

$\$$ Professor, College of Computing, 801 Atlantic Dr., 30332-0280.
} 
improved performance from systems exploiting molecular machinery, and atomically precise processing in space. Realizing this potential requires the development of atomically precise diamondoid and fullerene molecular machinery that can scale similar to the well-known biological molecular machinery of proteins, RNA, and DNA. This, in turn, requires satisfactory early progress in design and analysis, and continued progress to mature the capability. A number of challenges must be resolved before molecular nanotechnology can be applied to space exploration.

The first challenge is component design. The discrete nature of molecular components and the computationally intensive tools required to analyze them makes their design difficult. Multiplying the challenge, many useful systems will require a large number of distinct molecular components, necessitating automated design tools. We suggest an approach to move forward on this challenge under "IIA. Molecular Component Design" below.

The second challenge is reaction pathway design; given desired molecular components there must be some way to fabricate them. In order to achieve the flexibility required for the synthesis of molecular machine systems, novel synthetic methods involving positional assembly must be employed. This can be accomplished using molecular positional devices to control synthetic reaction trajectories. In early experimental work the positional devices may be scanning probe microscopes. Reaction pathway design must ultimately integrate with molecular component design. We suggest activities to move forward on this challenge under "IIB. Molecular Component Reaction Pathway Design" below.

The third challenge is design and realization of molecular fabrication systems capable of synthesizing molecular components in a useable form. This includes providing devices for positional control, defining the mechanosynthesis unit operation set, providing physical and information inputs for each unit operation, adequate parallelism, the system infrastructure, and molecular machinery supporting these core functions and overall utility. Mechanosynthetic methods and molecular machinery can be theoretically validated with high quality ab initio, density functional theory and molecular mechanics techniques, and must eventually be validated experimentally. We address this challenge under "IIIA. Fabrication System Design and Development" below.

The fourth and final challenge is integrating molecular machinery into the design and production of space exploration systems. This includes fabricating molecular components, assembly products at the appropriate level of convergent assembly, and integrating products into the final space exploration systems. We touch on the many facets of this challenge below under "IIC. Folding Molecular Component Design Into System Design," "IVA. Relative Timing of System Design to System Realization," "IIB. Molecular Component Development and Fabrication," and "IIIC. System Development and Realization."

Because molecular nanotechnology is a high-payoff enabler for robust space activities, but there are a great many uncertainties about how and when it will develop, weighty and difficult management issues are raised. We touch on these under "IV. Challenges in Development Timeline and Planning Uncertainty."

First we summarize previous work in how space systems may exploit molecular nanotechnology, and of relevant progress in molecular nanotechnology.

\section{A. Previous Work on the Potential of Molecular Nanotechnology for Space Exploration}

To a first approximation, any stable arrangement of atoms that can be described at a high level is a candidate design for a product of molecular nanotechnology. This has led to analysis based example conceptual designs using molecular nanotechnology performance parameters. Following several early works on the applicability of molecular nanotechnology for space exploration ${ }^{1-3}, \mathrm{M}^{\mathrm{c}}$ Kendree analyzed in detail ${ }^{4}$ a broad array of space system architectures and showed the potential for 1-4 orders of magnitude performance improvement over current technology, using molecular nanotechnology. Higher level analyses addressed other space system architectures such as extraterrestrial resource extraction and processing, and closed environment life support systems.

\section{System and Mission Benefits of Improved Structures}

One potential of molecular nanotechnology is to fabricate structural elements for space systems of very high strength-to-weight, including diamond compressive members, nanotube-based tension members, and composite structures of nanotube fibers in a diamond matrix. Diamond has a $\sigma$ of $5.0 \times 10^{10} \mathrm{~Pa}$ with a $\delta$ of $3510 \mathrm{~kg} / \mathrm{m}^{3}$, and thus a strength to density ratio $\sim 70$ times better than Titanium. Nanotubes have a $\sigma$ of $4.5 \times 10^{10} \mathrm{~Pa}^{5}$ with a $\delta$ of 1300 $\mathrm{kg} / \mathrm{m}^{3}$, and thus a strength-to-density ratio $\sim 2.4$ times better than diamond, but only in tension.

Such materials, if used to reduce parasitic rocket mass, can reduce rocket dry masses by $\sim 98 \%$ and thereby triple rocket payloads to Earth orbit. Depending on the cost model, this can improve launch costs by a factor somewhere between 3 and $235^{4}$. Towers that extended just above the Earth's atmosphere as efficient launch platforms ${ }^{6}$ could easily be supported with diamond or nanotube material ${ }^{4}$. A number of such towers could support a linear accelerator 
running at the edge of the atmosphere powered by terrestrial power-lines, creating a "space pier" for launching payload into orbit on the order of $\$ 1 / \mathrm{kg}^{\S \S}$.

If the tower extends high enough then a payload may climb the tower and will be in orbit when released. This has a standard analysis ${ }^{6-10}$, and is usually conceived as a structure tapered to keep stresses feasible and a cable in tension to improve structural stability. A mixed structure of an actively stabilized tower in compression reaching to a cable in tension is more mass efficient on Earth ${ }^{4}$. The total required mass of the structure is extremely sensitive to the strength-to-density ratio of the structural material. Nanotubes bring these "space elevators" into the realm of the structurally feasible, although additional issues must be resolved before they can be practical ${ }^{4,11}$.

While there are a variety of candidate concepts, and the preferred concept given molecular nanotechnology may not even be identified yet, the clear indication is that once the structural materials offered by molecular nanotechnology can be easily deployed in systems, some form of greatly reduced launch costs would become available. Launch costs in the $\$ 1-\$ 100$ per kg range would then open the solar system to much wider exploration and exploitation. There is a potential for conventional nanotechnology to make feasible and affordable nanotubebased structures sooner than molecular nanotechnology. If so, then many of the benefits described above can be implemented, providing launch cost savings in their own right, and acting as precursors to a more mature molecular nanotechnology.

If a tension structure is not tied to a planet, but instead freely rotates in space, then it can be much smaller, but still useful in providing a change in velocity $(\Delta \mathrm{V})$ to payloads with a "throw" or more challenging "catch." ${ }^{, 2,13}$ The improved strength-to-density of nanotubes means they can have nearly three times the tip speed of conventional materials and thus offer almost three times the $\Delta \mathrm{V}$, which is sufficient to reach most of the solar system from elliptic Earth orbit ${ }^{4}$.

Structures experience a slight force from absorbing and reflecting sunlight, and in space detectable accelerations are possible. A smaller mass per unit of light gives higher acceleration. Such structures should be useable for navigation in space as solar sails ${ }^{14}$. With molecular nanotechnology, those surfaces could be $20-100 \mathrm{~nm}$ thick sheets of aluminum able to provide $1-13 \mathrm{~km} / \mathrm{s}$ of $\Delta \mathrm{V}$ per day. Nets of metalicized nanotubes might also be feasible, and could offer accelerations of $17 \mathrm{~m} / \mathrm{s}^{2}$ or more ${ }^{15}$. Unfortunately, since solar sails cannot generate a sunward acceleration greater than solar gravity, trajectory shapes are limited, and the metalicized nanotube performance shaves only a few percent off typical travel times. ${ }^{4}$.

If a large, thin structure facing the Sun is the active layer of solar cells, then a specific power of $\sim 10 \mathrm{kWe} / \mathrm{kg}$ at 1 $\mathrm{AU}$ is feasible. Since vehicle performance using solar-electric ion engines scales linearly with the specific power of the power source, this offers roughly two orders of magnitude improvement in performance, which can be distributed between acceleration or exhaust velocity and $\Delta \mathrm{V} .^{4}$

The substantial performance in space transportation offered by molecular nanotechnology may make feasible or more efficient some level of long-duration interstellar transport.

2. System and Mission Benefits of Molecular Machinery

Another potential of molecular nanotechnology is to provide mechanical devices with part sizes down to molecular components. Excluding systems which use such devices to fabricate products by rearranging molecular structures, other application to space exploration has been identified.

Designs in Ref. 4 use tiny motors to individually steer reflective solar concentrators $0.1 \mathrm{~mm}$ in diameter. Their small size allows them to hold optic tolerance while presenting $3 \times 10^{-4} \mathrm{~kg} / \mathrm{m}^{2}$ of mass per unit area to the Sun, and this raises the specific power to $739 \mathrm{kWe} / \mathrm{kg}$, available for a further factor of 70 improvement in solar-electric ion engines and other purposes. At this level interplanetary trips can take weeks with reaction mass a minority of initial vehicle mass.

A number of molecular nanotechnology concepts for swarms of small robots have been proposed, and could be considered for use in space ${ }^{3}$.

Mechanical ${ }^{16}$ and other ${ }^{17}$ nanotechnology computers could be designed, and could be used in space vehicles. Their main on-board advantages are to provide very high memory densities ${ }^{18}$, high speed high device densities ${ }^{17}$, tiny avionics for tiny vehicles, or to provide computers with high thermodynamic efficiency for computation near the thermodynamic limit ${ }^{19}$. Autonomy would be a significant use of on-board computational resources. Off-board, high computing power could be used in very large amounts for system and detail design, including computationally intensive evolutionary design algorithms.

$\S \S$ Hall, J. S., “A Space Pier," International Space Development Conference 2005, 16 May 2005, URL: http://isdc2005.xisp.net/ kmiller/isdc_archive/fileDownload.php/?link=fileSelect\&file_id=162, [cited 30 July 2005]. 


\section{System and Mission Benefits of in situ Molecular Manufacturing}

A third potential of molecular nanotechnology is to provide systems of mechanical devices with part sizes down to molecular components to fabricate products by rearranging molecular structures.

The easiest case to consider is using molecular manufacturing to remanufacture material brought with the mission. One concept would be for a space vehicle to remanufacture itself, using the same mass to provide different subsystems at different times in support of different mission phases ${ }^{20}$.

A more challenging case is to take material brought that is no longer in the desired form, and remanufacturing into the desired form. One application would be a closed-environment life-support system (CELSS), rebuilding fresh air, water and food from wastes. Molecular manufacturing has high throughput efficiency, as most processing is done by small devices operating at high frequency. A molecular nanotechnology CELSS may mass less than $40 \mathrm{~kg}$ per person, and draw somewhere between $125 \mathrm{We}$ and 4,000 We per person ${ }^{4}$. While this mass is very small, it is overwhelmed in space by the per capita mass required for radiation shielding. A second application is self-repair of the system itself. This requires full redundancy across the self-repair subsystem so that any failure in the self-repair subsystem can itself be repaired. Such a system can have an average mission life many orders of magnitude longer than its component lives ${ }^{20}$. With self-repair, structure limited systems like tethers and space elevators can have lower safety factors and higher performance, while material wear limited systems, like designs that expose bare diamond or nanotubes to solar UV, are feasible with longer lives ${ }^{4}$.

An interesting case is using molecular manufacturing to build new system mass from material found in situ. As the specific compositions and material structure of space resources still require significant exploration and research, the details of systems to implement this case are necessarily more speculative. Given the focus of molecular nanotechnology on using the lighter elements from the period table, carbonaceous asteroids, and other volatile-rich bodies are attractive targets in the solar system for this concept. Given the many uncertainties, the doubling period for a molecular nanotechnology mine/factory on an asteroid might range anywhere from a day to a year, with production costs ranging from $\$ 15$ to $\$ 2 \times 10^{-10}$ per $\mathrm{kg}^{4}$. This would make material resources in space quite available, with issues of ownership and control as important challenges.

4. Applicability to NASA's Exploration Mission

These capabilities are well beyond current technology, and would greatly assist NASA's Space Exploration Mission objectives, as sketched in Table 1 below.

Table 1. Example applications of molecular nanotechnology to NASA's Exploration mission.

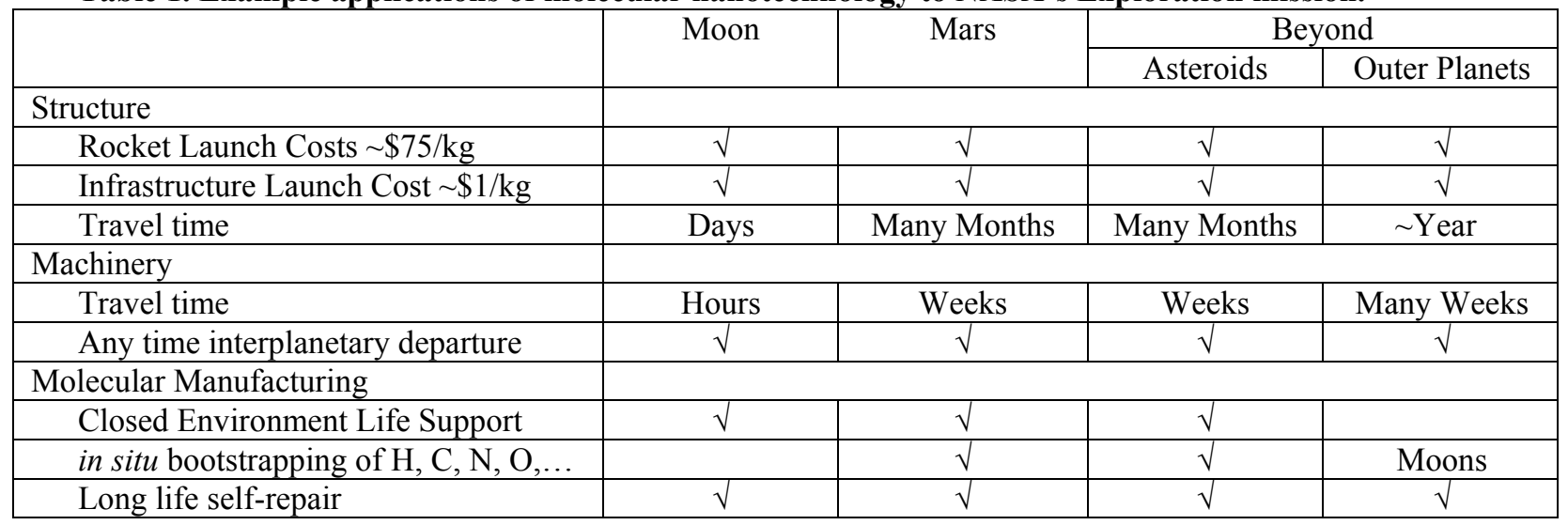

\section{B. Previous Molecular Nanotechnology Work}

The landmark experimental demonstration of positional atomic assembly occurred in 1989 when Eigler and Schweizer ${ }^{21}$ spelled out the IBM logo using 35 xenon atoms arranged on nickel surface using a scanning probe microscope (SPM). But molecular manufacturing requires bond formation. The use of precisely applied mechanical forces to induce site-specific chemical transformations is called mechanosynthesis. Oyabu et $\mathrm{al}^{22}$ achieved the first experimental demonstration of mechanosynthesis in 2002, using purely mechanical forces to make and break covalent bonds, first abstracting and then rebonding a single silicon atom to a silicon surface with SPM positional control at low temperature. 


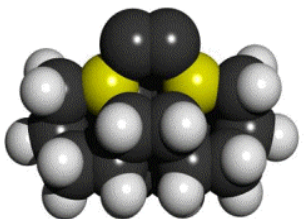

Figure 1. Merkle-Freitas Dimer deposition tool ${ }^{34}$, used with permission.

Package (VASP) with $\begin{gathered}\text { initio } \\ \text { generalized }\end{gathered}$ gimulation approximation.

In 1992, it was theorized ${ }^{16}$ that two nested carbon nanotubes would make an exceptionally low-friction nanomechanical bearing, then confirmed experimentally in 2000 by Cumings and Zettl ${ }^{38}$ at UC Berkeley. Using molecular mechanics force fields, Drexler and Merkle ${ }^{16}$ hand-designed several diamondoid molecular machines, some exclusively hydrogen and carbon atoms (Figure 3 on left) and others with additional elements such as silicon and oxygen (Figure 3 on right). Computational simulations of several of these devices by Goddard's group at CalTech ${ }^{39}$ confirmed the proper machine operation expected at $\mathrm{GHz}$ frequencies.
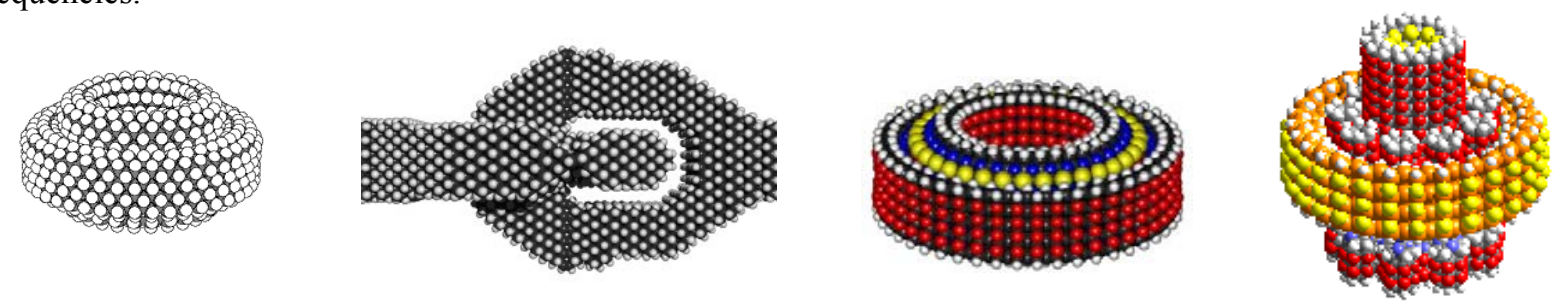

Figure 3. Various molecular device mechanical designs ${ }^{16}$, used with permission.

At NASA Ames Research Center (ARC), Han and colleagues ${ }^{40}$ designed and computationally tested a family of carbon nanotube based atomically-precise gears (Figure 4). The gear shaft consists of carbon nanotubes of different chiralities. The gear teeth are aromatic benzyne $\left(\mathrm{C}_{6} \mathrm{H}_{4}\right)$ bound to the nanotube. A similar known reaction occurs under mild conditions between other planar molecules such as naphthalene and anthracene $^{41}$, and fullerenes like $\mathrm{C}_{60}$. DFT predicts ${ }^{42}$ that o-benzyne attaches to the nanotube in the center of the six-membered rings, turning the nearby carbon atoms from $\mathrm{sp}^{2}$ to $\mathrm{sp}^{3}$ bonding. Molecular dynamics, using a parallelized version of Brenner's reactive carbon potential with a Leonard-Jones 6-12 potential for forces between molecules, was used to examine operating modes. Rotation at 50$100 \mathrm{GHz}$ was found to be optimal. At higher speeds the teeth slipped but remained intact. When the rotation rate was lowered again, interatomic forces returned the gears to perfect operating condition (unlike metal gears) - suggesting that atomic-scale gears are much more robust than their macroscopic counterparts.

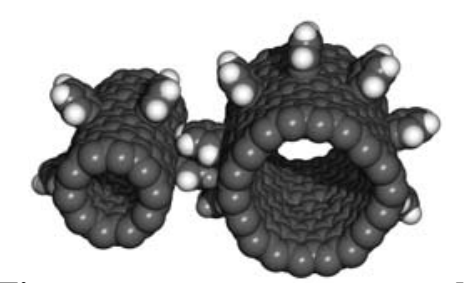

Figure 4. Nanotube shaft and benzyne ring gear design $^{40}$, Government work uncopyrighted figure.

\section{Challenges in Molecular Nanotechnology Design}

Molecular nanotechnology design is resolving the question of "What to do?" The specific molecular components for an application must be specified. That may eventually be via catalog, but developing the catalog requires design of specific molecular components. An effective reaction pathway for actually creating each of the desired molecular components must also be specified. Finally, the complete system design must be specified, including what is to be done with the desired molecular components. 
Very specific approaches are presented below for solving the subcomponent connector design problem, and for molecular component reaction pathway design. While these represent our best suggested approach under the current state of the art, with the ongoing march of science and technology alternative approaches may become more attractive in the future.

\section{A. Molecular Component Design}

A molecular component is a logically distinct, minimal assembly of atoms which goes into a larger system. Its design is fully specified by the isotope, positions and bonds of its atoms. For most purposes, stable isotopes of the same element are fully interchangeable in a molecular component design. A molecular component may be used to further build a larger assembly, and thus may have dangling bonds, but should be stable against rearrangement. As a detailed example, a potential solution to the Subcomponent Connector Design Problem is sketched out below.

The ability to connect subcomponents is fundamental to manufacturing. At the molecular scale, connecting two molecular subcomponents requires precise alignment of the relevant surface bonds, an issue not present when connecting macroscale components. If the two molecular subcomponents share identical crystal orientation (e.g., the subcomponents both share a common diamond C(110) surface) then the bonds from the two connecting faces could easily be aligned. More commonly, the two subcomponents will have differing complex surfaces or must be connected at an orientation that does not permit alignment of the surfaces. In this situation, an interfacial molecular connector is required to "fill the gap" between the two subcomponents.

Experience shows that hand design of such "gap filling" structures is very difficult and has seldom been done except in the simplest of cases. The use of automated methods to design appropriate connectors is therefore critical if we are to build more complex molecular machines by connecting subcomponents into components, and components into larger assemblies. Something as fundamental as connecting a bearing sleeve to a flat surface requires a complex gap-filling connector at the molecular scale.

One promising approach is using advanced automated algorithms to design molecularly precise components, starting from a set of disconnected diamond and fullerene subcomponents which are then joined together using molecular connectors. The methods, which include genetic algorithms, genetic programming, simulated annealing, and variants thereof, have recently begun to produce humancompetitive results in real-world application domains $^{43}$. For example, in NASA's Space Technology 5 (ST5) mission, a requirementscompliant evolved antenna (Figure 5A) was produced that is scheduled to be flown in 2005 - the first
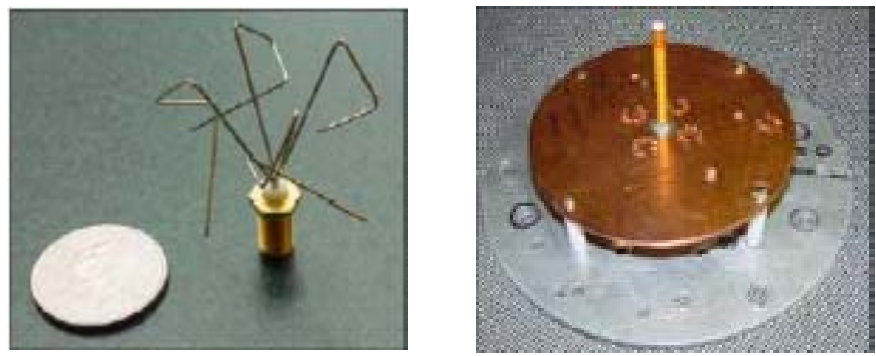

Figure 5. Antennas, Evolved Design on left (A) and Conventional Human Designed on right (B). evolved hardware ever flown on a NASA mission. The best evolved designs outperformed a conventional humandesigned model (Figure 5B) especially in critical metrics such as mass and cost.

Evolutionary algorithms are stochastic search methods that mimic the metaphor of natural biological evolution ${ }^{44}$. Evolutionary algorithms operate on a population of potential solutions applying the principle of survival of the fittest to produce better and better approximations to a solution. At each generation, a new set of approximations is created by the process of selecting individuals according to their level of fitness in the problem domain and breeding them together using operators borrowed from natural genetics. This process leads to the evolution of populations of individuals that are better suited to their environment than the individuals from which they were created.

Evolutionary algorithms model natural processes such as selection, recombination, mutation, migration, locality and neighborhood. Figure 6 shows the structure of a simple evolutionary algorithm for automated design. By operating on a population of solutions, evolutionary algorithms perform their search in parallel. To solve a component-design problem using evolutionary algorithms requires a representation for molecular subcomponent connectors, mutation and crossover operators, and a fitness function to evaluate individual designs.

One approach is to use genetic programming (GP), wherein an evolvable computer program automatically written in a special-purpose programming language is the representation genotype ${ }^{45}$. The program could then be executed to generate the molecular subcomponent connector design. The full molecular component design (subcomponents + connector) would minimized by a molecular force field and then evaluated for stiffness. Candidate existing force fields include the existing Brenner, Tersoff, Stillinger-Weber, Amber, Charmm, Gromos, UFF, Dreiding, MM2, and MM3 force fields. If a unique nanomechanical-specific force field were developed, that should provide greater confidence and accuracy. 


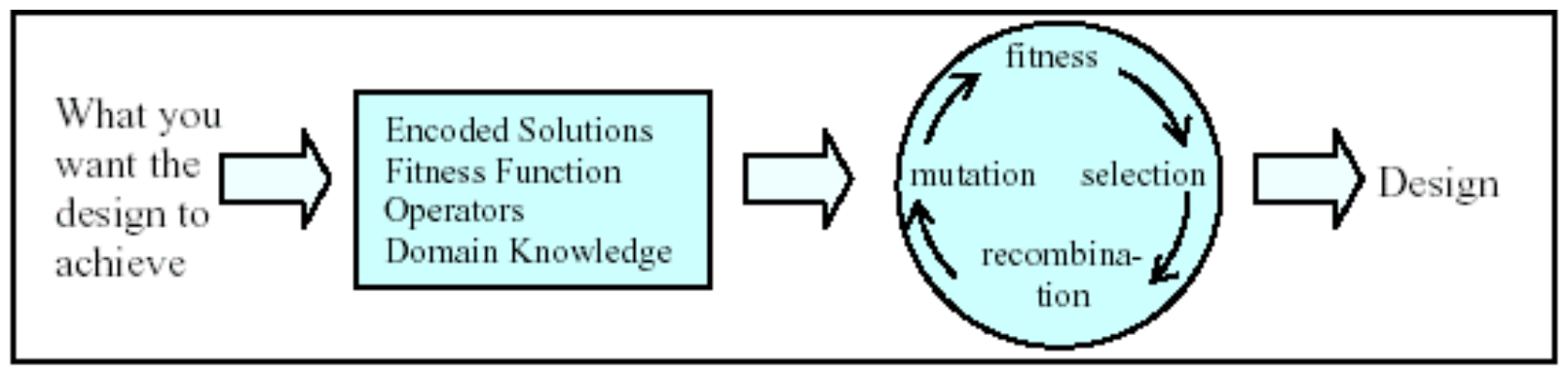

Figure 6. The algorithmic structure of evolutionary design, used with permission.

Such an evolving program can be represented by a tree, with nodes for constants, arithmetic operators, loops, subroutine calls, data structures (simple variables, arrays, lists, stacks, queues, etc.), conditionals, and primitive operations in the domain. Mutation and crossover are simple tree transformations. Koza ${ }^{46}$ also uses so-called structure-modification 'mutations'. These do not change the phenotype, but rather make equivalence transformations on the evolving program to introduce or remove subroutines, loops, and data structures. GP is credited with humanclass performance on a number of problems, including evolving pre-existing patents and even novel patentable inventions.

Koza's GP approach is designed for extreme generality. While this permits attacking new problems with minimal change to the algorithm, fitness functions for molecular connector designs include resource-hungry structural stiffness calculations mandating the fewest possible evaluations. Thus, extending Koza's GP representation to include object-oriented programming (OOP) constructs - classes, instance variables, inheritance, and dynamic binding - should take greater advantage of expected regularities in nanomechanical subcomponent connections. Also, more sophisticated data nodes could limit the range of variables to reduce the size of the search space. Finally, Koza's structure-modifying mutations do not change the phenotype, which is inefficient. Combining the structure-modifying mutations with functional mutations guaranteed to take advantage of new structural possibilities.

Constructing stiff subcomponent connectors is a poorly understood process, but solutions can be evaluated by computational chemistry techniques. Finding such solutions is a "black art", well-suited to evolutionary techniques. Evolution substitutes a very large number of inexpensive computerized cut-and-try operations for slow and expensive, albeit more intelligent, human analysis.

The corresponding input to evolution would be molecular component definitions consisting of two or more disconnected diamond and/or fullerene subcomponents located in three-space with allowable tolerances for subcomponent translational and rotational degrees of freedom. Permitted and forbidden regions for the molecular connectors could also be defined. The evolutionary problem is to connect the molecular subcomponents with a set of atoms covalently bound to the subcomponents and to each other. The primary evaluation criteria is stiffness, as measured by numerical derivatives calculated using the force field.

The procedure to be followed would include a number of primitive operations, including minimization with optional freezing of subcomponents, short molecular dynamics runs, inserting atoms, removing atoms, inserting diamond, inserting graphite, inserting n-membered rings, inserting cages, hydrogen termination of dangling bonds, and other similar operations. The primitive operations to apply, their parameters (e.g., minimization termination criteria, atom placement, etc.), and the order of application will be discovered by evolution.

The fitness function is multi-objective, combining connector stiffness, residual strain, number of atoms, computation time, and other factors. Stiffness will be calculated by using the force field to produce numerical derivatives for each of the six degrees of freedom for each input-specified molecular subcomponent. The measure of residual strain will be the energy per atom relative to bulk materials. The computation time should be measured, with very long-running procedures cut off.

A first useful step would be to develop the ability to evolve molecular connectors between two specified diamond and/or a limited set of fullerene molecular subcomponents with very tight tolerances using a minimal set of construction primitives. Once achieved, the technique could extend to three or more molecular subcomponents, loosen the tolerance bounds, expand the range of allowed subcomponent types, increase the number of construction primitives, and improve the force field. The optimal candidate molecular systems produced via genetic programming and molecular force fields should be tested and validated using scalable parallel ab initio quantum chemistry codes. These codes compute accurate single point energies as well as analytic gradients (i.e., forces) for 
systems containing a few hundred to more than 1000 atoms. If the computed forces are too large, then the particular candidate is still too far from equilibrium and it should be rejected.

It should be noted that successful artificial evolution of solutions to difficult problems such as the subcomponent connection problem is dependent on large computational resources. Fortunately, computation is bounded by the large number of independent phenotype generation and fitness function evaluations required. This is a significantly parallel computational problem ideally suited to large parallel machines, such as NASA ARC's 10,000-node Columbia $^{* * *}$.

\section{High Value Component Validation}

One can determine molecular forces and properties, including component stiffness and residual strain, using the most reliable and scalable Multiconfiguration Self Consistent Field (MCSCF) code in General Atomic and Molecular Electronic Structure System (GAMESS) ${ }^{47-49}$ that has demonstrated high efficiency on more than 1,000 processors of the Origin SSI architecture. MCSCF gives a versatile and general description of electron correlation effects through its CI formulation. The parallel MCSCF code was developed at NASA ARC by Graham Fletcher. Predicting bulk properties of larger systems, however, will require the development of methods with greater size and computational scalability. The SCF level of theory will span RHF (closed-shell), ROHF (open-shell), and UHF wave functions as needed. A replicated data SCF algorithm can be used for calculations on systems containing a few hundred atoms. For larger problems a code will need improved scalability. For example, a technique in which larger calculations are initiated using the superposed charge densities of smaller subcomponent systems should scale better. In this way, accurate wave functions for increasingly large systems could be generated with great efficiency, since each calculation builds upon the previous ones.

To facilitate calculations on systems with 1000 atoms or more, a distributed data algorithm will be necessary. With distributed data algorithms, not only are calculations faster, but larger calculations become possible as more processors are added. In this sense, the speedup is unlimited. Times-to-solution will simultaneously reflect the memory and processor speed improvements of supercomputing. A reasonable goal to target would be demonstrating at least an $80 \%$ parallel efficiency benchmark on 2,048 CPUs.

\section{B. Molecular Component Reaction Pathway Design}

Specific reactions required for the synthesis of molecular components must be developed and validated. In order to achieve the flexibility required for the synthesis of molecular machine systems, novel synthetic methods involving positional assembly (e.g., the use of scanning probe microscopes or molecular positional devices to control synthetic reaction trajectories) must be employed. These novel synthetic methods, and the molecular machine systems that will use them, can validated with high quality ab initio, density functional theory and molecular mechanics techniques. Due both to their strong aerospace benefits as high strength-to-mass materials ${ }^{4}$, and their great flexibility as engineered molecular components ${ }^{16}$, diamondoid, fullerene and related structures are the most attractive targets.

\section{Reaction Pathways for Diamond/Fullerene Synthesis}

The elucidation of specific theoretical reaction pathways are required to build the evolved molecularly precise components, from molecular primitives, via site-specific chemical reactions made possible by techniques of positionally-controlled mechanosynthesis enabled by SPMs or new positional devices. The elucidation of these pathways can be based on an efficient combination of lower-precision DFT computations on larger numbers of atoms (e.g., several hundreds), using the Vienna Ab initio Simulation Package (VASP) and comparable software, and of high-accuracy quantum ab initio computations on smaller numbers of atoms (e.g., several tens), using PSI ${ }^{50}$,

$\mathrm{Q}^{-C_{h} \mathrm{~m}^{51}}{ }^{\text {, MOLPRO }}{ }^{\dagger \dagger}$, GAMESS ${ }^{47}$, and/or comparable programs. Such simulations can be run on large, highspeed multiprocessor cluster computers, including the Columbia 10,000-node computer at NASA ARC. Synthetic targets include carbon nanotubes and diamond.

\footnotetext{
****NAS Project: Columbia," URL: http://www.nas.nasa.gov/About/Projects/Columbia/columbia.html [cited 27 July 2005].

iti "MOLPRO, a package of ab initio programs designed by H.-J. Werner and P. J. Knowles." Version 2002.6. H.-J. Werner, P.J. Knowles, M. Schütz, R. Lindh, P. Celani, T. Korona, G. Rauhut, R.D. Amos, A. Bernhardsson, A. Berning, D.L. Cooper, M.J.O. Deegan, A.J. Dobbyn, F. Eckert, C. Hampel, G. Hetzer, A.W. Lloyd, S.J. McNicholas, F.R. Manby, W. Meyer, M.E. Mura, A. Kicklass, P. Palmieri, R. Pitzer, U. Schumann, H. Stoll, A.J. Stone, R. Tarroni, and T. Thorsteinsson. URL: http://rs2.ch.liv.ac.uk/dlc/MOLPRO.html [cited 27 July 2005]
} 


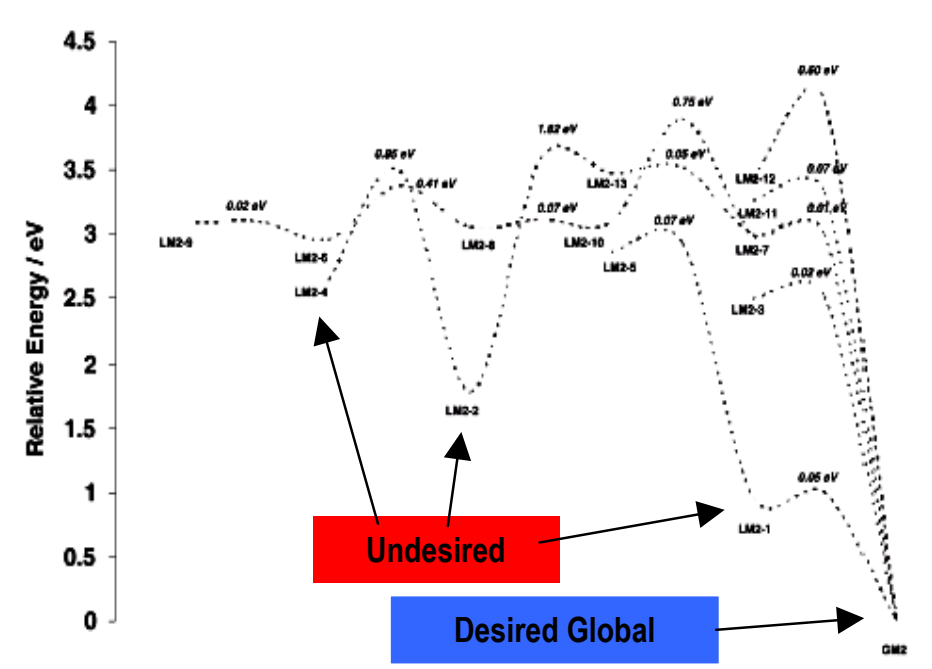

Figure 7. Energetics of $\mathrm{CC}$ dimer placements on the diamond C(110) surface $^{35}$, used with permission.

Early efforts should specify validated models of relevant surfaces, then evaluate possible surface reconstructions, thermal desorption events, and surface diffusion kinetics of hydrogen and carbon moieties. One can then analyze the potential energy minima for various conformations of carbon dimers bonded to diamond surface via site-specific mechanosynthesis, to determine the required positional placement accuracy needed to build the desired diamond lattice while avoiding numerous undesired local minima (LM) representing defect formations. This approach extends on work already done by Peng, Freitas and Merkle ${ }^{35,36}$ for CC dimer placements on the diamond $\mathrm{C}(110)$ surface (Figure 7). A parallel early target is the initial development and analysis of validated tools for diamond and carbon nanotube mechanosynthesis, including tools for hydrogen abstraction, hydrogen donation, and carbon placement on diamond surface, work already initiated by Freitas and Merkle ${ }^{52}$.

Such early work would lay the foundation to complete the development and analysis of validated tools for diamond and nanotube mechanosynthesis. This will ultimately require expanding the toolset to include (1) a carbon abstraction tool, and (2) additional non-HC tools as required by evolved-component analysis. A critical related study would be of the thermal uncertainty of positional placement for extended tooltip handle designs, to verify that the designed tools, when actually deployed in an experimental setting, will perform with sufficient placement accuracy to ensure defect-free construction of diamond lattice. Also building on the earlier work once could then design and test tooltip recycling pathways, using the existing toolset in combination with bulk regeneration processes, with the objective of moving toward a fully self-contained, minimal rechargeable diamond mechanosynthetic toolset. Another approach to consider is parts assembly via fusion of similar surfaces, both of diamond and nanotubes.

\section{Modeling Strategy}

To support reaction pathway design, a multi-scale modeling approach should be pursued, in which rigorous quantum mechanical methods are applied to small model systems to calibrate more approximate force-field models which may be applied to much larger systems. This would leverage expertise in computational materials science and high-accuracy $a b$ initio quantum chemistry. It should be stressed that existing force-field and density functional theory models have difficulty in accurately describing bond-breaking reactions, and hence calibration (and possible re-parameterization) by comparison against high-quality, correlated ab initio methods is necessary to put the modeling efforts on a firm footing.

\section{Model Structure Building}

Reliable structure models for $\mathrm{C}(111), \mathrm{C}(110)$ (Figure 8) and $\mathrm{C}(100)$ surfaces are necessary prerequisites for mechanosynthesis. In initial efforts, one may employ a combined approach using density functional theory (DFT) and classical molecular dynamics (MD) to create different surfaces. The classical MD would use force fields to be obtained from DFT calculations (and validated against higher-accuracy ab initio computations on small cluster models) $)^{53,54}$. The desired surface structure can be searched and optimized with classical MD. Either pure DFT or DFT MD can be employed using the structures from the classical MD as input. This approach can be very effective to obtain large scale structures which have complex and longtime structure relaxation. With further optimization, the same approach can

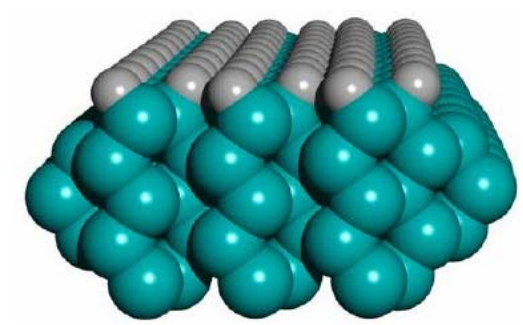

Figure 8. Model of the $\mathbf{C}(110)$ diamond surface.

be used to obtain surfaces with steps and hexagonal diamond (lonsdaleite) surface. As an illustration of feasible analytic size, the in-house computational capability of co-author Li's group can comfortably handle calculations on 100-500 C atoms using VASP ${ }^{55}$. In parallel with the bulk diamond models, small cluster models should be developed to be used in high-accuracy ab initio benchmark computations of reaction pathways. 


\section{High-Accuracy ab initio Methods}

When used in conjunction with large basis sets, wavefunction-based electronic structure methods with extensive treatments of electron correlation (e.g., many-body perturbation theory and coupled-cluster theory) have been shown to give very reliable predictions of molecular properties, approaching the reliability of experiment in some cases ${ }^{56}$. Moreover, as rather sophisticated, parameter-free approximations to the electronic Schrödinger equation, these methods are applicable in principle to any chemical system, including any of the structures and processes proposed here. Unfortunately, however, achieving the great accuracy of these methods requires a very unfavorable scaling with system size $\left[\mathrm{O}\left(\mathrm{N}^{7}\right)\right.$ for the typical "gold standard" method, coupled-cluster with perturbative triples ${ }^{57}$, $\operatorname{CCSD}(\mathrm{T})]$. Hence, $a b$ initio computations should be performed on small model systems to validate the approximations to be used for larger-scale modeling. In particular, $a b$ initio results can help assess which functionals are best for use in the medium-size computations (up to a few hundred atoms), and in the development of force field models for much larger simulations.

Although the equilibrium structures of diamond surfaces or clusters are not especially challenging computationally, reaction pathways and thermodynamics are not as straightforward, and the use of appropriatelychosen ab initio models becomes very important for obtaining reliable results and for calibrating the DFT and force field models. Ab initio methods can be used to compute the thermodynamics of the abstraction and placement reactions, as well as possible recycling pathways. These methods can also be used to provide barrier heights for surface migration simulations. Bond-breaking and bond-making reactions can be difficult to model because they often involve electronic near-degeneracies which are not well described by the standard methods ${ }^{58-68}$. By comparing to high-level benchmarks evaluated with multi-reference methods \{multi-reference configuration interaction, multiconfigurational self-consistent-field (MCSCF), or complete-active-space second-order perturbation theory (CASPT2)\}, one can determine which of the more typical $a b$ initio methods are suitable for prototype reactions. To make this determination, it should be useful to also evaluate various DFT methods and parameterize force field models. These taxing multi-reference computations may be made easier by the recent discovery that minimal active spaces actually perform as well as larger active spaces for bond-breaking reactions when compared to exact (full CI) benchmark results ${ }^{68}$. Extensions of Krylov's spin-flip methods, recently developed by the Sherrill group in collaboration with Krylov ${ }^{60,64}$, may also be helpful.

\section{Force Fields $(F F)$}

As mentioned above, one goal of the ab initio calculations is to obtain reliable force fields for $\mathrm{C}-\mathrm{C}, \mathrm{C}-\mathrm{H}$, and $\mathrm{C}$ 1,2-mer interactions, with the objective of extending the calculation to large scales and of accelerating the entire computing process (see below). There are three cases where large-scale calculations are requisite for mechanosynthesis: (1) Although previous works have demonstrated preliminary feasibility and reaction pathways for diamond mechanosynthesis, most of these studies used fairly small models $(<100 \mathrm{C} \text { atoms })^{32}$, and it remains unknown whether the process will be affected in a larger molecular environment. (2) In certain circumstances, carbon nanotubes or large pieces of diamond must be fused to create larger systems, a process requiring classical MD using FF. (3) Elevated temperatures must be considered in mechanosynthesis. Besides using DFT MD, classical $\mathrm{MD}$ with FF is a viable alternative. There is no ideal, off-the-shelf solution to obtain the FF, so this requires a new or custom-developed program. To improve accuracy and confidence, one should use not only data from bulk samples but also data from surface properties and from high-accuracy quantum calculations on small clusters to fit the FF.

6. DFT MD and Kinetic Monte Carlo Calculations

Zero-temperature calculation usually ignores thermal, vibrational and other dynamic effects on various processes in mechanosynthesis. These effects are important factors in determining the feasibility of mechanosynthesis. In searching for mechanosynthetic reaction paths one can employ molecular dynamics along with DFT calculations, allowing one to obtain thermal and dynamic responses. Using the energetic and barrier information provided from high procession calculations, one can also carry out kinetic Monte Carlo calculations for possible surface reconstructions, thermal desorption events, and surface diffusion kinetics of hydrogen and carbon moieties. The comprehensive nature of such analysis requires a collaborative, multiscale, and multi-level calculation approach that would press beyond the current state of the art.

\section{Folding Molecular Component Design Into System Design}

Another key challenge is integrating molecular machinery into the design of space systems. Conceptually this is straightforward; one need merely follow the hierarchy where subsystems at one level are considered systems with their own subsystems at the next lower level, and continue down until reaching the level of molecular components as systems with atoms as subsystems. We will refer to these in the arbitrary scale case as modules comprising components. 
One difficulty is design scope, which can be handled by design reuse. Each $\mathrm{kg}$ of final system may have over $10^{25}$ atoms, and if molecular components typically comprise $\sim 100$ atoms then each of $\sim 10^{23}$ molecular components in a $\mathrm{kg}$ could in principle be unique. Specifying such detail is daunting. Through design re-use, however, standardized modules at each level of scale vastly reduce the total description size of an end-user system. In this approach catalog modules are defined as particular arrangements of catalog components. Atoms are the components at the lowest level. A key parameter for tractability is the average number of non-catalog components per module across the levels of design scale. Developers of new real designs should strive to drive this towards 1.0, because the total design complexity will explode as this parameter grows above 1.

While the top-level of an aerospace system design and even some key components could be human-specified, the vast part count means that large swaths of the detail design must be automatically generated. This means that the final, executable design must be machine-readable.

To generate the catalog, module candidates should be generated by bottom-up analysis, computationally validated, and ultimately demonstration. Beyond the actual design description, the functional, performance and other characteristics of molecular components and larger modules should be captured, so these results can flow into higher-level module and system design. A design pull from efforts at higher levels to create representative useful designs of larger modules can help guide the catalog generation to useful regions of the module design-space.

To ease Design For Manufacturability, the module catalog for design should indicate catalog modules for assembly, and design should use such modules wherever possible. For a desired new system design, the default approach would be top-down design and specification to particular molecular components. Once a catalog design part is selected, the entire physical decomposition is fully specified, and once a catalog assembly part is specified, the default manufacturing flow is fully specified. If a catalog design part is selected that is not a catalog assembly part, then some translation into physical manufacturing control is needed. Provision of such an algorithm is a requirement for a non-assembly design part to be included in a fully useful design catalog. An interesting class of such parts is ones that are to be manufactured by first assembling catalog modules, and then rearranging or removing selected atoms that would ordinarily remain. The inclusion of this class of design module breaks the convergent assembly approach wherein modules larger than molecular components are fabricating only by assembly, which simplifies manufacturing planning.

Beyond a catalog of specific modules, it would also be useful to have algorithms that generate executable designs of interesting classes of products. For example, an algorithm that takes as an input an arbitrary volume description and generates the design of a diamond with terminated surfaces that fills the volume. A more challenging example would fill the arbitrary volume with a nanotube fiber-diamond matrix composite material, giving the fibers a desired volume fraction, fractal dimension, and anisotropicity. Large repeating units for these algorithm-generated structures would be excellent catalog modules, but some will probably require non-catalog molecular components, and thus algorithms for generating molecular component designs.

Evolutionary algorithms can be used for generating module designs. Performed off-line, this would support the design of catalog modules. Performed in-line with system design, which could generate bespoke modules, mechanisms and components for special-use, without necessarily requiring human intervention, but could delay the system design process.

\section{Challenges in Development and Implementation of Molecular Nanotechnology}

Molecular nanotechnology development and implementation is both answering the question "How to do it," and actually doing it, which must be resolved before integrating molecular machinery into the production of space systems. This includes fabricating molecular components, assembly products at the appropriate level of convergent assembly, and integrating products into the final space exploration systems

\section{A. Fabrication System Design and Development}

Molecular nanotechnology requires systems that can synthesize molecular components and deliver them in a useable form, either assembled into a final product or as or assembled into modules that other manufacturing systems can use to assemble into a final product. Such fabrication systems have only conceptual designs, and will require significant effort and new research to develop.

A key open research area is inventing, demonstrating, and maturing to high reliability devices for positional control of reactive moieties for site-specific mechanosynthesis. Early research can focus on SPM-controlled tooltips, but small, high-frequency devices are the desired end-state. Work must go beyond analysis of concepts such as robot arms and molecular mills ${ }^{16}$ to demonstration of actual devices, followed by detailed characterization, analysis 
and improvement of actual positional control devices. Specific quirks, limitations or characteristics of these devices must be properly handled by the fabrication system architectures.

Those architectures must be taken to detailed design. One such architecture is the "assembler" approach, wherein small platforms floating in solution with input molecules contain robotic mechanosynthesis arms used to fabricate small products in solution ${ }^{69-73}$. A more rigid such fabrication system architecture is the convergent factory model $^{16,73}$, where molecular mills create most or all molecular components and their first tiers of assembly, and tiers of robots flexibly assemble these into larger modules until fabricating the product. A third such architecture is the parts-fabricator/parts-assembler architecture ${ }^{73-75}$, which uses a combination of fabricators that make smaller modules and assemblers that combine modules into larger assemblies. It may be that the preferred architecture remains to be invented.

On the other hand, these are all analyzed examples of kinematic self-replicating machines (KSRM) ${ }^{73}$, which provide a general solution to the initial bootstrap problem. The large potential flexibility of a molecular fabrication system using mature molecular nanotechnology suggests that the range of products may include massively parallel and/or self-replicating nanofactories or molecular assemblers. The comprehensive 137-dimensional map of the kinematic replicator design space by Freitas and Merkle ${ }^{73}$ captures all of the above architectures, and likely much more.

Crucial questions in the field are defining the mechanosynthesis unit operation set and the mechanosynthesis tool set. A complete set of reactions and mechanosynthetic tools must be developed and validated. These molecular tools would then be used to synthesize molecular machines, including tools and molecular machines able to manufacture more tools and molecular machines. A key goal is developing a mechanosynthesis unit operation set that can produce all the molecular components necessary to build a fabrication system that has the same or larger unit operation set-this is the basis for one solution to the initial bootstrap problem. Molecular machinery design techniques must also be developed, including but not limited to interactive tools and automated evolutionary software methods. For aerospace systems using molecular nanotechnology, diamondoid components are very attractive. Published papers have analyzed a few reactions that could be used in the positional synthesis of diamondoid components, with Ref. 76 pushing towards a complete unit operation set. Most recently, Freitas and Merkle $^{52}$ have analyzed a minimal toolset consisting of three primary tooltypes composed solely of $\mathrm{C} / \mathrm{Ge} / \mathrm{H}$ atoms, intended for use in positionally-controlled diamond mechanosynthesis in a vacuum (UHV) environment, that appears capable of recharging and fabricating all three tooltypes as well as fabricating all three principal diamond surfaces, handle structures, 1D carbon and hydrocarbon chains, and single-walled carbon nanotubes. A more complete set of such reactions and mechanosynthetic tools must be computationally developed and validated. Finally, the tool-set must be implemented and integrated with actual positional devices.

A small, high-frequency device adding a million carbon atoms a second would take over a trillion years to build one gram of product. The only way to fabricate macroscopic quantities of product is with massive parallelism. This requires scaleable designs that can implement somewhere in the range of $10^{12}$ to $10^{26}$ simultaneous devices. This level of parallelism ensures some number of failed components, so the manufacturing system must also be able to work around a reasonable rate of failed components.

Given tools under positional control that implement a sufficiently wide set of mechanosynthethic unit operations, it remains necessary to provide the correct control, so that each work station implements the correct operation making the proper next step in the synthesis of the desired molecular component in effective service of building the desired product. This is closely tied to the selection of positional control device. Certain positional devices, like molecular mill may only implement a single unit operation. Other devices, like robot arms, may be able to select from many tools and implement a great many operations. Still other devices implement an intermediate or small number of operations, but with some choice that requires direction. The entropy of the worksite must be managed, requiring some combination of physical constraints that limit the feasible variability and sensing with detects variations and allows for corrective action. Worksite control ties to the architecture, and will drive the detailed system layout.

Also driving the detailed system layout are the infrastructure subsystems. Control is not the only thing that must flow in and out. Power must be distributed to the individual devices, and active cooling may be needed. Material flow must bring input feedstock to initial worksites, carry intermediate products to their next worksite, and carry away physical waste. The worksites must each be held together with sufficiently tight tolerances, and all must be held in place in their correct relative locations.

Finally, it is necessary to find and implement some development path. An element of the development path problem is the initial bootstrap problem. Somehow, sufficient parallelism of devices to make mole quantities of molecular components is needed. Early fabrication systems could be inherently massively parallel, for example if they are self-assembled in solution out of engineered proteins mass produced by biotechnology. If early fabrication 
systems are less parallel, however, then some way of bootstrapping more capacity from the early systems is needed. If the assembler approach is used, directly self-replication of an initial assembler can through exponential growth produce enough assemblers for adequate parallelism. A different initial architecture may require some path of early limited systems making more capable fabrication systems, which make more capable fabrication systems, which ultimately make a system of sufficient size and parallelism to build useful products. To follow such a path requires finding it.

\section{B. Molecular Component Fabrication}

Molecular component design is the special case of System design at the lowest module level, as discussed above in "IIC. Folding Molecular Component Design Into System Design." Given the design and the fabrication system it will remain to actually fabricate the molecular components. Manufacturing (i.e., the fabrication system) will need to be qualified. Even if the fabrication system is highly flexible, it will need to be qualified in demonstrating fabrication of the specified molecular components. Even if the fabrication system is highly reliable, it will need to be tested extensively before that reliability will be trusted. The molecular components will need to be output in a manner that can be used in System realization. Ordinarily that would be the components assembled into the largest module of direct use in system realization of which the fabrication system is capable (e.g., a complete payload box ready to be mounted on a satellite). In some special case, it may be the raw molecular components themselves, for example if there were a new aerospace thermoplastic.

\section{System Development and Realization}

Given a system design including molecular components, and a supply of those components, the design remains to be realized. The system design must be fully detailed and converted to realization instructions, primarily for manufacturing. Molecular component fabrication will assemble products using molecular components to the appropriate level of convergent assembly, and those products will need to be integrated products into the final space exploration system. It may be that the appropriate level of convergent assembly is the complete space system. For space systems, "realization" may well include launch and travel to targets in space, and other mission activities.

While the temptation is to assume that since the system design process is so detailed it will necessarily generate an ideal design, this is far from guaranteed. The requirements specified in may be in error. Limitations of the initial space system architecture, the system development process, or the fabrication system may produce less than ideal results. Also, since molecular manufacturing should reduce realization costs and cycle times ${ }^{130 l d}$, it may favor a more iterative design approach, with frequent prototyping and testing.

\section{Challenges in Development Timeline and Planning Uncertainty}

It is of little use to say dramatic new capabilities may be available some day if one cannot also provide some realistic sense that these capabilities may be available within some relevant planning horizon.

\section{A. Relative Timing of System Design to System Realization}

System design conducted long before system realization is exploratory engineering ${ }^{130 l d}$ or concept exploration. This is less concerned with getting the "right" system design than with getting a reasonable, very high confidence system design, in order to get a feel for concepts and their future capabilities. Because the design is not intended to be the final design, it is not necessary to work out in full detail.

System design conducted in parallel with key implementation development is concurrent engineering. If the system can be successful without molecular nanotechnology, but a change in design to incorporate new, atomically precise components would improve system success, this situation calls for opportunity management. If a system requires molecular nanotechnology but its life cycle is initiated before that is available, this situation calls for risk management. Fortunately, NASA has a well-established approach to handling emerging capabilities, based on Technology Readiness Levels (TRLs), and can handle this transition period less badly than many other organizations in many other domains.

Long traditional concepts of system design implicitly assume the component technologies are mature and demonstrated before the system design is initiated. Once molecular nanotechnology is demonstrated and mature, it will easily fit into aerospace system design.

\section{B. Planning For Long Durations with the Large Schedule Uncertainty of Molecular Nanotechnology}

Planning over a short term is relatively easy. Planning in the face of little uncertainty is relatively easy. NASA must make plans, however, that could impact Human Exploration of Mars in 2030. In addition, the schedule 
uncertainties around molecular nanotechnology are such that it could be unavailable in 2030, or the capabilities discussed in IA. above may start emerging in 2020. Planning over such uncertainty is daunting.

The minimal strategy is to ignore molecular nanotechnology until such time as its development appears likely within the time horizon of decisions being made. This would be an effective strategy for many purposes, but one cannot assure that the probability of molecular nanotechnology being developed and deployed before 2030 is low. This is especially true if one tries to weight the probability by the impact.

The classic formal strategy for decision under uncertainty ${ }^{77}$ is to build a decision tree and assess the probability, utility, and thus expected utility of various end-states given various decisions combined with random events. To do that for NASA in this case requires some modeling of the operational state of NASA's Exploration Mission circa 2030, given deployment of molecular nanotechnology for space exploration some years earlier. This is likely to require extrapolation of utilities beyond the range of ordinary NASA analyses, and thus will require some effort.

The concepts surrounding expected utility are well understood, and folded into aerospace management. Another strategy is simply to make sure that molecular nanotechnology is fully incorporated as subjects in the relevant management tools, such as technology portfolio development, risk management, and opportunity management. As an example high risk/high payoff opportunity for NASA Exploration Missions after Human return to the Moon by 2020, molecular nanotechnology is a compelling test case for longer-duration opportunity management. If the existing tools cannot well address this case, then they may need to be extended to adequately handle this and other outliers.

When decision trees are built in situations where there is a high driving uncertainty a significant period in the future, the typical result is that the preferred strategy is to invest in the near term to understand and better characterize that uncertainty, and only then make more consequential decisions. Even simpler than a decision tree analysis is to just pursue the initial strategy of characterizing the likely impacts and schedule uncertainty of molecular nanotechnology for space. Preliminary analyses of impacts exist. Understanding schedule uncertainty calls for an effort to develop a roadmap of possible ways to develop molecular nanotechnology for aerospace, and then assessing component schedule uncertainties and developing signposts for detecting when the schedule may be coming near enough to justify further action.

A final strategy is to decide that while the schedule is uncertain, the payoff is quite large, and thus pursue development of molecular nanotechnology, with the aim of accelerating the schedule without having to know what the schedule necessarily is. This may be an appropriate strategy for some organizations, but NASA's Exploration Mission Directorate has been given a Schedule-driven mission ("Return to the Moon by 2020"), not an event-driven mission ("Return to the Moon when it is cheap enough.").

In parallel with the basic approach, parallel activities should be able to capitalize on favorable developments. For example, NASA's science missions could increase their emphasis on the detailed characterization and cataloguing of resources in Space, which would be of great use whenever high capacity, low cost space operations become feasible, via molecular nanotechnology or other means.

\section{Conclusion}

In January 2004, NASA established a long-term program to extend human presence across the solar system, a primary goal of which will be to establish a human presence on the Moon no later than 2020, as a precursor to human exploration of Mars. These future NASA Exploration Missions can be completely transformed by molecular nanotechnology. NASA's Exploration Missions ultimately depend on the ability to manufacture specific structures with specific capabilities. At the present time, manufacturing systems are unable to build large products with molecular precision. Such ability would have a transformative system-of-systems impact on all human and robotic Exploration Missions. Performance, safety, sustainability, affordability, modularity and reliability can be radically improved, in many cases by orders of magnitude. Precise definition of molecularly-precise positionally-controlled molecular manufacturing systems, along with computational validation of feasibility, is suggested before such systems can be developed. Critical to this goal is computational development of aerospace-relevant molecular machinery processes and designs, particularly diamondoid- and fullerene-based computer systems, sensors, and actuators. These components will enable high-precision molecular manufacturing of propulsion and launch systems, thermal management systems, life support and consumables recycling, space energy production and storage, in situ resource processing, photon and magnetic sails, tethers, nanomechanical structures, radiation resistant computation, and self-repairing materials. Such end systems can be fielded once tools are developed for designing the necessary components of molecular devices, and those components are designed, fabricated and assembled into appropriate devices and subsystems, and then the subsystems are integrated into space systems. Several of these challenges can 
be directly addressed immediately, including progress in molecular component design and molecular component reaction pathway design, and further concept exploration on aerospace systems and missions that would exploit molecular nanotechnology. Other challenges will need to be addressed indirectly or over time. While this areas offers great promise, only when such systems are created and used will the potential of molecular nanotechnology for space exploration be realized.

\section{Acknowledgments}

We wish to thank encouragement, suggestions and review to a prior draft by Graham Fletcher and Jason Lohn.

\section{References}

${ }^{1}$ Drexler, K.E., "Molecular Manufacturing for Space Systems: An Overview," Journal of the British Interplanetary Society, Vol. 45 No. 10, 1992, pp. 401-405

${ }^{2} \mathrm{M}^{\mathrm{c}}$ Kendree T. L., "Implications of Molecular Nanotechnology: Technical Performance Parameters on Previously Defined Space System Architectures," Nanotechnology, Vol. 7, No. 3, 1996, pp. 204-209.

${ }^{3}$ Globus A., Bailey D., Han J., Jaffe R., Levit C., Merkle R. C., and Srivastava D., "NASA Applications of Molecular Nanotechnology," Journal of the British Interplanetary Society, Vol. 51, 1998, pp. 145-152.

${ }^{4} \mathrm{M}^{\mathrm{c}}$ Kendree, T. L., "A Technical and Operational Assessment of Molecular Nanotechnology for Space Operations," Ph.D. Dissertation, Industrial and Systems Engineering Dept., Univ. S. Cal., Los Angles, CA, 2001.

${ }^{5}$ Walters D. A., Ericson L. M., Casavant M. J., Liu J., Colbert D. T., Smith K. A., Smalley R. E. "Elastic Strain of Freely Suspended Single-Wall Carbon Nanotube Ropes." Applied Physics Letters, Vol. 74, No. 25, 1999, pp 3803-3805.

${ }^{6}$ Landis G. A., Cafarelli, C. "The Tsiolkovski Tower Re-Examined," Journal of the British Interplanetary Society. Vol. 52, 1999, pp. 175-180.

${ }^{7}$ Lvov V., "Sky-Hook: Old Idea," Science, Vol. 158, 1967, pp. 946-947.

${ }^{8}$ Isaacs J. D., Vine A. C., Bradner H., Bachus G.E. "Satellite Elongation Into a True 'Sky-Hook',” Science, Vol. 151, 1966, pp. 682-683.

${ }^{9}$ Pearson J., "The Orbital Tower: A Spacecraft Launcher Using the Earth's Rotational Energy," Acta Astronautica, Vol. 2, No. 9-10, 1975, pp. 785-799.

${ }^{10}$ Logsdon T., Orbital Mechanics: Theory and Applications, John Wiley \& Sons, New York, 1998, pp. $242-248$. 2003.

${ }^{11}$ Edwards B. C., Westling E. A., The Space Elevator: A Revolutionary Earth-to-Space Transportation System, Edwards BC,

${ }^{12}$ Moravec H. P., “A Non-Synchronous Orbital Skyhook,” Journal of the Astronautical Sciences, No. 25, No. 4, 1977, pp. 307-322.

${ }^{13}$ Hoyt, R. P., Uphoff, C., "Cislunar Tether Transport System," 35th AIAA/ASME/SAE/ASEE Joint Propulsion Conference \& Exhibit, AIAA-99-2690, 1999.

${ }^{14}$ McInnes C. R., Solar Sailing: Technology, Dynamics and Mission Applications, Springer-Verlag in association with Praxis Publishing, Chichester, UK, 1999.

${ }^{15}$ Christensen, C. "Appendix A-Nanogrid Reflectance Equations," Ultra Thin Solar Sails for Interstellar Travel: Phase I Final Report, Speith, D., Zubrin, R., Pioneer Astronautics Inc., Lakewood, Colorado, 1999, pp. 16-30.

${ }^{16}$ Drexler K. E., Nanosystems: Molecular Machinery, Manufacturing, and Computation, John Wiley \& Sons, New York, 1992.

${ }^{17}$ Ellenbogen J. C., Love J. C., Architecture for Molecular Electronic Computers: 1. Logic Structures and an Adder Built From Molecular Electronic Diodes, MP 98W0000183, MITRE, McLean Virginia, 1999.

${ }^{18}$ Bauschlicher C. W. Jr., Ricca A., R. C. Merkle, "Chemical Storage of Data," Nanotechnology, Vol. 8, No. 1, 1997, pp. 1-5.

${ }^{19}$ Merkle R. C., Drexler K.E., "Helical Logic,” Nanotechnology, Vol. 7, No. 4, 1996, pp. 325-339.

${ }^{20} \mathrm{M}^{\mathrm{c} K e n d r e e ~ T . ~ L ., ~ " T h e ~ L o g i c a l ~ C o r e ~ A r c h i t e c t u r e, " ~ N a n o t e c h n o l o g y, ~ V o l . ~ 9, ~ N o . ~ 3, ~ 1998, ~ p p . ~ 212-222 . ~}$

${ }^{21}$ Eigler D. M., Schweizer E. K., "Positioning Single Atoms with a Scanning Tunneling Microscope," Nature, Vol. 344, 1990, pp. 524-526.

${ }^{22}$ Oyabu N., Custance O., Yi I., Sugawara Y., Morita1 S., "Mechanical Vertical Manipulation of Selected Single Atoms by Soft Nanoindentation Using Near Contact Atomic Force Microscopy," Physics Review Letters, Vol. 90, 2003, p. 176102.

${ }^{23}$ Page M., Brenner D. W., "Hydrogen Abstraction from a Diamond Surface: Ab Initio Quantum Chemical Study Using Constrained Isobutane as a Model," Journal of the American Chemical Society, Vol. 113, 1991, pp. 3270-3274.

${ }^{24}$ Sinnott S. B., Colton R. J., White C. T., Brenner D. W., "Surface Patterning by Atomically-Controlled Chemical Forces: Molecular Dynamics Simulations," Surface Science, Vol. 316, 1994, pp. L1055-L1060.

${ }^{25}$ Brenner D. W., Sinnott S. B., Harrison J. A., Shenderova O. A., "Simulated Engineering of Nanostructures," Nanotechnology, Vol. 7, No. 3, 1996, pp. 161-167.

${ }^{26}$ Musgrave C. B., Perry J. K., Merkle R. C., Goddard W. A. III, "Theoretical Studies of a Hydrogen Abstraction Tool for Nanotechnology," Nanotechnology, Vol. 2, No. 4, 1991, pp. 187-195.

${ }^{27}$ Chang X. Y., Perry M., Peploski J., Thompson D. L., Raff L. M., "Theoretical Studies of Hydrogen-Abstraction Reactions from Diamond and Diamond-Like Surfaces,” Journal of Chemical Physics, Vol. 99, 1993, pp. 4748-4758. 
${ }^{28}$ Lyding J. W., Hess K., Abeln G. C., Thompson D. S., Moore J. S., Hersam M. C., Foley E. T., Lee J., Chen Z., Hwang S. T., Choi H., Avouris P. H., Kizilyalli I. C., "UHV-STM Nanofabrication and Hydrogen/Deuterium Desorption from Silicon Surfaces: Implications for CMOS Technology," Applied Surface Science, Vol. 130, 1998, 221-230.

${ }^{29}$ Foley E. T., Kam A. F., Lyding J. W., Avouris P. H., "Cryogenic UHV-STM Study of Hydrogen and Deuterium Desorption from Si(100)," Physics Review Letters, Vol. 80, 1998, pp. 1336-1339.

${ }^{30}$ Hersam M. C., Abeln G. C., Lyding J. W., "An Approach for Efficiently Locating and Electrically Contacting Nanostructures Fabricated via UHV-STM Lithography on Si(100)," Microelectronic Engineering, Vol. 47, 1999, pp. $235-237$.

${ }^{31}$ Ricca A., Bauschlicher C. W. Jr., Kang J.K., Musgrave C.B., "Hydrogen Abstraction From a Diamond (111) Surface in a Uniform Electric Field,” Surface Science, Vol. 429, No. 1-3, 1999, pp. 199-205.

${ }^{32}$ Walch S. P., Merkle R. C., "Theoretical Studies of Diamond Mechanosynthesis Reactions,” Nanotechnology, Vol. 9, No. 3, 1998, pp. 285-296.

${ }^{33}$ Dzegilenko F. N., Srivastava D., Saini S., "Simulations of Carbon Nanotube Tip Assisted Mechano-Chemical Reactions on a Diamond Surface," Nanotechnology, Vol. 9, No 4, 1998, pp. 325-330.

${ }^{34}$ Merkle R. C., Freitas R. A. Jr., "Theoretical Analysis of a Carbon-Carbon Dimer Placement Tool for Diamond Mechanosynthesis," Journal of Nanoscience and Nanotechnology, Vol. 3, No. 3, 2003, pp. 319-324.

${ }^{35}$ Peng J., Freitas R. A. Jr., Merkle R. C., "Theoretical Analysis of Diamond Mechanosynthesis. Part I. Stability of $\mathrm{C}_{2}$ Mediated Growth of Nanocrystalline Diamond C(110) Surface," Journal of Computational Theoretical Nanoscience, Vol. 1, 2004, pp. 62-70.

${ }^{36}$ D. J. Mann, Peng J., Freitas R. A. Jr., Merkle R. C., "Theoretical Analysis of Diamond Mechanosynthesis. Part II. C 2 Mediated Growth of Diamond C(110) surface via Si/Ge-Triadamantane Dimer Placement Tools," Journal of Computational Theoretical Nanoscience, Vol. 1, 2004, pp. 71-80.

${ }^{37}$ Peng J., Freitas R. A. Jr., Merkle R. C., Von Ehr J. R., Randall J. N., Skidmore G. D., "Theoretical Analysis of Diamond Mechanosynthesis. Part III. Positional C2 Deposition on Diamond C(110) Surface Using Si/Ge/Sn-based Dimer Placement Tools," Journal of Computational Theoretical Nanoscience, (unpublished).

${ }^{38}$ Cumings J., Zettl A., "Low-Friction Nanoscale Linear Bearing Realized from Multiwall Carbon Nanotubes," Science Vol. 289, No. 5479, 2000, pp. 602-604.

${ }^{39}$ Cagin T., Jaramillo-Botero A., Gao G., Goddard W.A. III, "Molecular Mechanics and Molecular Dynamics Analysis of Drexler-Merkle Gears and Neon Pump," Nanotechnology, Vol. 9, No. 3, 1998, pp. 143-152.

${ }^{40}$ Han J., Globus A., Jaffe R., Deardorff G., "Molecular Dynamics Simulation of Carbon Nanotube Based Gears," Nanotechnology, Vol. 8, No. 3, 1997, pp. 95-102.

${ }^{41}$ Hoffmann R.W., Dehydrobenzene and Cycloalkynes, Academic Press, New York, 1967.

${ }^{42}$ Jaffe R., Han J., Globus A., "Formation of Carbon Nanotube Based Gears: Quantum Chemistry and Molecular Dynamics Simulations of the Electrophilic Addition of o-Benzyne to Fullerenes, Graphene, and Nanotubes," First Electronic Molecular Modeling \& Graphics Society Conference, , online conference, 1996.

${ }^{43}$ Koza J. R., Keane M. A., and Streeter M. J., "What's AI Done for Me Lately? Genetic Programming's Human-Competitive Results," IEEE Intelligent Systems, Vol. 18, May/June 2003, pp. 25-31.

${ }^{44}$ Goldberg, D. E., Genetic Algorithms in Search, Optimization and Machine Learning, Addison-Wesley, New York, 1989.

${ }^{45}$ Koza J. R., Genetic Programming: on the Programming of Computers by Means of Natural Selection, MIT Press, Cambridge, 1992.

${ }^{46}$ Koza J. R., Bennett F. H., Keane M. A., Genetic Programming III: Darwinian Invention and Problem Solving, ISBN: 1558605436, Morgan Kaufmann Publishers, San Francisco, 1999.

${ }^{47}$ Schmidt M. W., Baldridge K. K., Boatz J. A., Elbert S.T., Gordon M. S., Jensen J. J., Koseki S., Matsunaga N., Nguyen K. A., Su S., Windus T. L., Dupuis M., Montgomery J. A. Jr., "General Atomic and Molecular Electronic Structure System (GAMESS)," Journal of Computational Chemistry, Vol. 14, 1993, pp. 1347-1363.

${ }^{48}$ Fletcher G. D., Gordon M. S., Schmidt M. W., "Developments in Parallel Electronic Structure Theory," Advanaced Chemical Physics, Vol. 110, 1999, p. 267.

${ }^{49}$ Fletcher G. D., Gordon M. S., Schmidt M. W., "Developments in Parallel Electronic Structure Theory," Computational Physics Communications, Vol. 128, 2000, p. 190.

${ }^{50}$ Crawford T. D., Sherrill C. D., Valeev E. F., Fermann J. T., King R. A., Leininger M. L., Brown S. T., Janssen C. L., Seidl E. T., Kenny J. P., Allen W. D., "PSI 3: An Open-Source Ab Initio Electronic Structure Package," Journal of Computational Chemistry (submitted for publication).

${ }^{51}$ Kong J., White C. A., Krylov A. I., Sherrill C. D., Adamson R. D., Furlani T. R., Lee M. S., Lee A. M., Gwaltney S. R., Adams T. R., Daschel H., Zhang W., Korambath P. P., Ochsenfeld C., Gilbert A. T. B., Kedziora G. S., Maurice D. R., Nair N., Shao Y., Besley N. A., Maslen P. E., Dombroski J. P., Baker J., Byrd E. F. C., Van Voorhis T., Oumi M., Hirata S., Hsu C.-P., Ishikawa N., Florian J., Warshel A., Johnson B. G., Gill P. M. W., Head-Gordon M., Pople J. A., Journal of Computational Chemistry, Vol. 21, 2000, pp. 1532-1548.

${ }^{52}$ Freitas R. A. Jr., Merkle R. C., "A Minimal Toolset for Positional Diamond Mechanosynthesis," Journal of Computational Theoretical Nanoscience, (unpublished).

${ }^{53}$ M. Li, Goddard III W.A., "Phenomenological Many-Body Potential From Interstitial Electron Model," Journal of Chemical Physics, Vol. 98, 1993, p. 7995.

${ }^{54}$ Li M., Goddard III W. A., "Interstitial Electron Model for Lattice Dynamics in Metals," Physical Review B, Vol. 46, 1992, p. 5237. 
${ }^{55}$ Ulundogan M., Li M., "Violation of Stokes-Einstein Relation in Equilibrium Eutectic CuZr Liquid: an Ab Initio Molecular Dynamics Calculation," Physics Review Letters, (to be published).

${ }^{56}$ Lee T. J., Scuseria G. E., “Achieving Chemical Accuracy With Coupled-Cluster Theory,” Quantum Mechanical Electronic Structure Calculations with Chemical Accuracy, edited by Langhoff S. R., Kluwer, Dordrecht, 1995, pp. 47-108.

${ }^{57}$ Raghavachari K., Trucks G. W., Pople J. A., Head-Gordon M., "A 5th-Order Perturbation Comparison of Electron Correlation Theories," Chemical Physics Letters, Vol. 157, 1989, pp. 479-483.

${ }^{58}$ Hirao K. (Editor), Recent Advances in Multireference Methods: Volume IV of Recent Advances in Computational Chemistry, World Scientific, New Jersey, 1999.

${ }^{59}$ Sherrill C. D., "Bond Breaking in Quantum Chemistry," Annual Reports in Computational Chemistry, Volume I, Spellmeyer D., editor, Elsevier, Amsterdam, 2005, pp. 45-54.

${ }^{60}$ Krylov A. I., Sherrill C. D., "Perturbative Corrections to the Equation-of-Motion Spin-Flip SCF Model: Application to

Bond-Breaking and Equilibrium Properties of Diradicals," Journal of Chemical Physics, Vol. 116, 2002, pp. $3194-3203$.

${ }^{61}$ Hoffmann M. R., Sherrill C. D., Leininger M. L., Schaefer H. F., "Optimization of MCSCF Excited States Using Directions of Negative Curvature," Chemical Physics Letters, Vol. 355, 2002, pp. 183-192.

${ }^{62}$ Abrams M. L., Sherrill C. D., "A Comparison Of Polarized Double-Zeta Basis Sets and Natural Orbitals for Full Configuration Interaction Benchmarks," Journal of Chemical Physics, Vol. 118, 2003, pp. 1604-09.

${ }^{63}$ Dutta A., Sherrill C. D., "Full Configuration Interaction Potential Energy Curves for Breaking Bonds to Hydrogen: An Assessment of Single-Reference Correlation Methods," Journal of Chemical Physics, Vol. 118, 2003, pp. 1610-1619.

${ }^{64}$ Sears J. S., Sherrill C. D., Krylov A. I., "A Spin-Complete Version of the Spin-Flip Approach to Bond Breaking: What Is the Impact of Obtaining Spin Eigenfunctions?” Journal of Chemical Physics, Vol. 118, 2003, pp. 9084-9094.

${ }^{65}$ Abrams M. L., Sherrill C. D., "An Assessment of The Accuracy of Multireference Configuration Interaction (MRCI) and Complete-Active-Space Second-Order Perturbation Theory (CASPT2) for Breaking Bonds to Hydrogen," Journal of Physical Chemistry Part A, Vol. 107, 2003, pp. 5611-5616.

${ }^{66}$ Abrams M. L., Sherrill C. D., "Natural Orbitals as Substitutes for Optimized Orbitals in Complete Active Space Wavefunctions," Chemical Physics Letters, Vol. 395, 2004, pp. 227-232.

${ }^{67}$ Abrams M. L., Sherrill C. D., "Full Configuration Interaction Potential Energy Curves for the X $1 \Sigma g+$, B $1 \Delta \mathrm{g}$, and B' $1 \Sigma \mathrm{g}+$ States of $\mathrm{C}_{2}$ : A Challenge for Approximate Methods Journal of Chemical Physics, Vol. 121, 2004, pp. 9211-9219.

${ }^{68}$ Sears J. S., Sherrill C. D., "On the Choice of Reference in Multi-Reference Electronic Structure Theory: Minimal References for Bond Breaking," Molecular Physics, Vol. 103, 2005, pp. 803-814.

${ }^{69}$ Merkle R. C., "Design Considerations for an Assembler," Nanotechnology, Vol. 7, No. 3, 1996, pp. 210-215.

${ }^{70}$ Merkle R. C., "Binding Sites for a Simple Assembler," Nanotechnology, Vol. 8, No. 1, 1997, pp. 23-28.

${ }^{71}$ Merkle R. C., “A New Family of Six Degree of Freedom Positional Devices,” Nanotechnology, Vol. 8, No. 2, 1997, pp. 4752.

${ }^{72}$ Merkle R. C., “Casing an Assembler,” Nanotechnology, Vol. 10, No. 3, 1999, pp. 315-322.

${ }^{73}$ Freitas R. A. Jr., Merkle R. C., Kinematic Self-Replicating Machines, Landes Bioscience, Georgetown, Texas, 2004, chaps. $4,5$.

${ }^{74}$ Freitas R. A. Jr., Gilbreath W. P., Advanced Automation for Space Missions, NASA Conference Publications CP-2255 (N83-15348), National Technical Information Service, Springfield, Virginia, 1982, chap. 5.

${ }^{75}$ Hall J. S., "Architecture Considerations for Self-Replicating Manufacturing Systems," Nanotechnology, Vol. 10, No. 3, 1999, pp. 323-330.

${ }^{76}$ Merkle R. C., “A Proposed 'Metabolism' for a Hydrocarbon Assembler,” Nanotechnology, Vol. 8, No. 2, 1997, pp. 149-162.

${ }^{77}$ Raiffa H., Decision Analysis: Introductory Lectures on Choices Under Uncertainty, McGraw Hill, New York, 1968. 